\title{
Video Conferencing Evaluation Considering Scalable Video Coding and SDN Network
}

\author{
Avaliação de Vídeo Conferência Considerando Codificação de Vídeo Escalável e Rede \\ SDN
}

\author{
Francisco Oliveira ${ }^{1}$, Eduardo Tavares $^{1 *}$, Erica Sousa ${ }^{2}$, Bruno Nogueira $^{2}$
}

\begin{abstract}
Video conferencing is very common nowadays, and it may contemplate heterogenous devices (e.g., smartphones, notebooks, game consoles) and networks in the same session. Developing video conferencing systems for this myriad of devices with different capabilities requires special attention from system designer. Scalable video coding (SVC) is a prominent option to mitigate this heterogeneity issue, but traditional Internet protocol (IP) networks may not fully benefit from such a technology. In contrast, software-defined networking (SDN) may allow better utilization of SVC and improvements on video conferencing components. This paper evaluates the performance of video conferencing systems adopting SVC, SDN and ordinary IP networks, taking into account throughput, delay and peak signal-to-noise ratio (PSNR) as the metrics of interest. The experiments are based on Mininet framework and distinct network infrastructures are also considered. Results indicate SDN with SVC may deliver better video quality with reduced delay and increased throughput.
\end{abstract}

Keywords: SDN - Video conferencing - Scalabe video coding - PSNR - Mininet

Resumo: Atualmente, vídeo conferência é bastante popular e pode contemplar dispositivos (e.g., smartphones, notebooks, vídeo games) e redes de computadores heterogêneas na mesma sessão. O desenvolvimento de sistemas de vídeo conferência para estes dispositivos com diferentes capacidades requer atenção especial de um projetista. Codificação de vídeo escalável (SVC) é uma opção proeminente para lidar com os problemas de heterogeneidade, mas as redes tradicionais que adoptam o protocolo da Internet (IP) podem não se beneficiar totalmente dos recursos da tecnologia SVC. Entretanto, redes definidas por software (SDN) podem utilizar os vídeos SVC de forma mais apropriada, permitindo uma melhoria nos componentes dos sistemas de vídeo conferência. Este artigo avalia o desempenho de sistemas de vídeo conferência considerando SVC, SDN e redes tradicionais IP, adotando as métricas vazão, atraso e relação sinal-ruído de pico (peak signal-to-noise ratio - PSNR). Os experimentos são baseados no framework Mininet e infraestruturas de redes distintas também são levadas em consideradas. Os resultados experimentais apontam que SDN com SVC podem contribuir em uma melhor qualidade de vídeo com menor atrasado e maior vazão.

Palavras-Chave: SDN, Vídeo conferência - Codificação de vídeo escalável - PSNR - Mininet

${ }^{1}$ Center of Informatics, Federal University of Pernambuco, Recife, Pernambuco, Brazil

${ }^{2}$ Department of Statistics and Informatics, Federal Rural University of Pernambuco, Recife, Brazil

*Corresponding author: eagt@cin.ufpe.br

DOI: http://dx.doi.org/10.22456/2175-2745.79310 • Received: 01/01/2018 • Accepted: 28/03/2018

CC BY-NC-ND 4.0 - This work is licensed under a Creative Commons Attribution-NonCommercial-NoDerivatives 4.0 International License.

\section{Introduction}

Over the last decades, video conference has become very popular due to better Internet acessibility, devices with more capabilities (e.g. faster CPUs) and free services (e.g., Google Hangout) [1]. A prominent trend indicates the growth of video conference services for desktop and mobile devices [1], which will be higher in 2020 than similar services based on rooms with expensive and sophisticated equipment.

Such an environment change creates new challenges, as not all clients have the same computational power, proper screen resolution, and similar bandwidth. Indeed, the heterogeneity of users is a remarkable issue. For instance, multipoint control unit (MCU) [2] is usually adopted to connect the devices on a video conference, and the heterogeneity of users imposes a great resource usage from MCU to transcode 
specific videos for distinct devices. Besides, most video conferencing systems adopt traditional IP networks, which are impacted by variations on bandwidth, delay and packet losses. Attempts to deal with these quality of service (QoS) issues, such as integrated (IntServ) and differentiated services (DiffServ), have not been fully successful due to the distributed architecture of the Internet [3].

To deal with limitations of traditional IP networks, new proposals for the Internet of the Future have been presented, and software-defined networking (SDN) is a representative technology [4]. SDN separates the control and data plane of switches/routers, such that the network control logic can be placed on an external entity and switches/routers (data plane) are only responsible for packet forwarding. Therefore, SDN allows the creation of a single view abstraction for the entire network, allowing great flexibility in network design and management [5]. Openflow [6] is the first successful protocol for the communication between data and the control planes.

Scalable video coding (SVC or H.264/SVC) is a standard to overcome device heterogeneity problem [7], in which a single video is encoded in multiple layers containing distinct features regarding spatial resolution, frame rate and quality. These layers are organized in different packets that can be transmitted separately to the same destination. There is a dependence among the layers. The base layer is the most important, since it is responsible for the generation of upper layers (that have better quality). As a consequence, SVC may considerably simplify the transcoding for MCUs. For instance, in the case of slower devices, upper layers may be discarded and only the base layer is transmitted. Multiversion coding is another proposal to deal with device heterogeneity, in which different video streams are transmitted simultaneously (simulcast) [8]. However, there are issues, for instance, related to larger bandwidth utilization in comparison to SVC.

Advances in video conferencing systems have taken into account distinct network and video technologies to mitigate the issues related to device heterogeneity and traditional IP networks. The adoption of different technologies motivates the performance evaluation of new systems, such as the impact of SDN network for prioritizing flows based on video conference. However, few works are available in the literature regarding performance evaluation of video conferencing systems with SDN and SVC (e.g., [9, 10]).

This work presents a performance evaluation of video conferencing systems, considering delay, throughput and peak signal-to-noise ration (PSNR) as the metrics of interest. Additionally, the impact of the following factors are assessed: (i) network type (traditional IP and SDN networks); (ii) and video type (non-scalable video and SVC). The experiments are based on Mininet framework, and they also take into account infrastructures with single and multiple paths. Results indicate SDN with SVC may deliver better video quality with reduced delay and increased throughput.

The remainder of this paper is organized as follows. Sec- tion II presents related work and Section III describes important concepts associated with this work. Section IV explains the adopted methodology. Section V details experimental results and, finally, Section VI concludes this work.

\section{Related Work}

Over the years, many techniques have been proposed for video conferencing systems and this section describes representative works that deal with device heterogeneity, QoS and quality of experience (QoE).

McCanne et al. [11] propose an adaptive algorithm based on layering encoding, in which the receiver is responsible for adapting the transmission rate according to its bandwidth constraint. Experimental results demonstate user-perceived quality is improved. Xu et al.[8] evaluate popular video conference services (Google Hangout, Skype, iChat) regarding system architecture, video encoding and adaptation technique. All services utilize a MCU-based approach and they adopt multiversion or multiple layers (i.e, SVC) for video encoding. Experiment results take into account real traffic and synthetic workload, and they show important features of each service. Additionally, layered video coding (e.g., Google Hangout) better deals with device heterogeneity due to lower overheads, and the authors state that prioritized selective retransmissions with SVC can improve even more user experience.

Castellanoes et al.[12] present a simulation framework for evaluating systems based on SVC, taking into account network congestion. Experiments are carried out and they demonstrate the impact of congestion on video quality (using PSNR). In [13] and [14], the authors propose frameworks for evaluating video conferencing and SVC systems, respectively, and they present experiments adopting wireless networks.

Egilmez et al. [3] propose a SDN controller for dealing with multimedia traffic considering QoS suport. Experimental results are presented using HTTP adaptive video streaming. Ongaro et al. [15] present an architecture for SDN networks to improve quality of service and real-time issues. Zhao et al. [9] also propose an architecture for multiparty video conferencing using a specialized media controller for SDN networks. Results demonstrate SDN can provide a better video delivery than traditional video conferencing architectures. Yang et al. [10] present a SDN architecture to deal with SVC multicast streaming. Experimental results show that bandwidth usage is reduced and QoE is not significantly affected. In that work, QoE is indirectly assessed using packet losses.

As demonstrated in the aforementioned works, SDN and SVC may provide improvements on video conferencing systems. However, a proper comparison is still required for pointing out situations in which each technology can outperform traditional, video conferencing technologies. This paper carries out a performance evaluation of video conferencing systems, taking into account the following factors: (i) network (traditonal, SDN); and (ii) video (non-scalable, SVC). We adopt throughput, delay and PSNR as the metrics of interest, which are utilized by representative works to assess video 
conferencing services. Besides, the proposed experiments also consider singlepath and multipath infrastructures.

Table 1 depicts a comparison of each related work (including this manuscript), taking into account SDN, SVC and performance evaluation (Performance) issues. Yang et al. [10] also carry out a performance evaluation considering SDN and SVC. However, their evaluation focuses only on the proposed architecture and do not execute further experiments, for instance, to assess distinct network infrastructures or the interaction between video and network technology (e.g., SVC in a non-SDN network). Besides, our work adopts PSNR to objectively assess QoE in the experiments.

\section{Background}

This section presents important concepts for a better understanding of this work. Firstly, video conferencing systems are introduced, followed by SDN networks and SVC.

\subsection{Video conferencing systems}

Video conferencing is an interactive communication system with the aim of enabling real-time meetings among three or more remote users, usually contemplating audio, video and even text. These systems usually require high bandwidth and low delay [9].

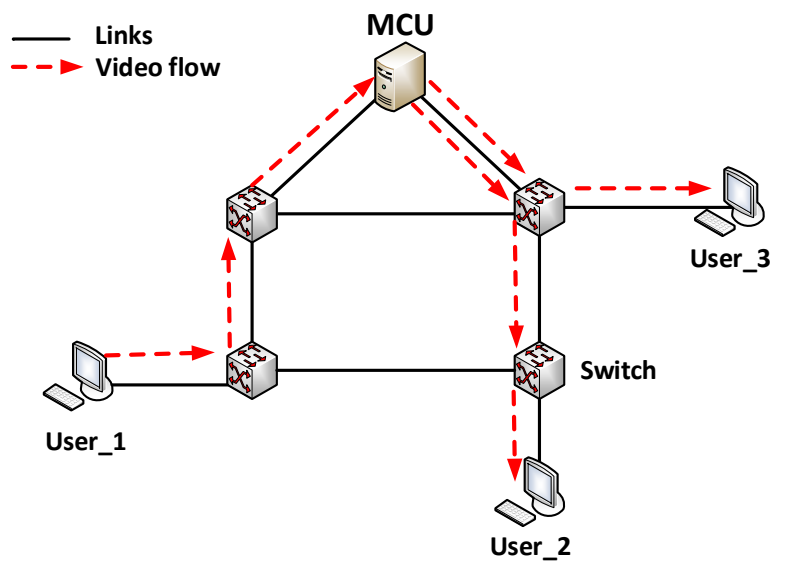

Figure 1. Video conferencing system

Figure 1 depicts a traditional video conferencing system adopting a multipoint control unit (MCU). The latter is commonly utlized to allow multiple devices in a conference, and it is responsible for managing the video traffic. For instance, each participant sends its video to MCU, which further converts the video considering each device capability (transcoding) and, then, transmit the converted videos to each conference member using distinct connections.

Transcoding is carried out whenever the video needs to be modified to meet the requirements of other participants. Mixing is another important functionality performed by MCUs, since it reduces several user connections only one. Since MCU considerably reduces the processing tasks for conference users, this unit may require many processing resources, which may limit the number of users that the system can support [16].

\subsection{Scalable Video Coding}

Scalable video coding is an extension of H.264/MPEG-4 advanced video coding (AVC) [17] and it considerably simplifies the adaptability required in many applications, for instance, to deal with network congestion. SVC adopts multiple layers organized in hierarchical arrangement (Figure 2), which encodes the video in a base layer (I) with low quality and additional layers, namely enhancement layers (B), with more data for improving video details. During a video streaming, the client can decode the video whenever the base layer is received, but video quality can be enhanced when upper layers are received. Despite the multilayer mechanism, SVC has similar encoding efficiency and decoding complexity as AVC [18].

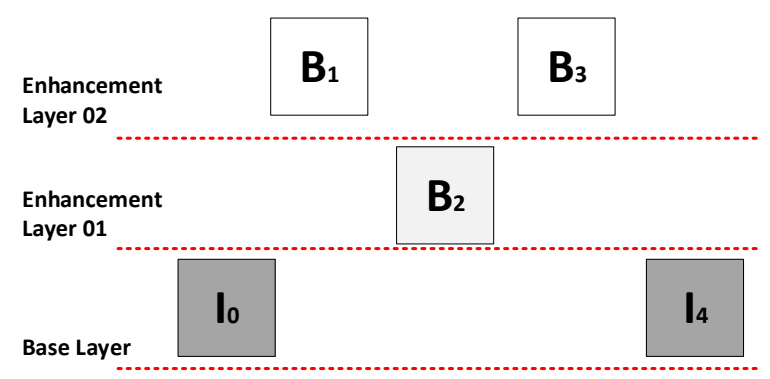

Figure 2. SVC streaming

Due to device heterogeneity, SVC provides good scalability for video conferencing systems. For instance, simulcast is the traditional mechanism for dealing with heterogeneity, which consists in transmitting various independent versions of the same video for different users. SVC considerably reduces the number of connections to transport different versions of the same video, since only one version with multiple layers is necessary. Besides, SVC can provide greater benefits in intelligent networks that adopt a prioritization mechanism, in the sense the packets related to the base layer can be prioritized in order to increase resilience against concurent traffic during the conference.

\subsection{Software-Defined Networking (SDN)}

Internet has provided an important platform for the creation of prominent distributed services, mostly based on client-server model, and, in this case, a static network archictecture was sufficient to meet service requirements. However, over the years, new services (based on cloud computing, for instance) have changed the traffic pattern, which requires a dynamic operation mode on Internet networks [5]. SDN is a representative technology that deals with this issue. 
Table 1. Comparison table of related works

\begin{tabular}{c|c|c|c|l}
\hline Work & Scalable Video & SDN & Performance & \multicolumn{1}{|c}{ Contribution } \\
\hline McCanne et al. [11] & $\checkmark$ & & & Adaptive algorithm based on the receiver \\
Xu et al. [8] & $\checkmark$ & & $\checkmark$ & Evaluation of video conference services \\
Castellanos et al.[12] & $\checkmark$ & & $\checkmark$ & Simulation framework \\
Klaue et al. [13] & $\checkmark$ & & $\checkmark$ & Evaluation framework \\
Detti et al.[14] & $\checkmark$ & & $\checkmark$ & Evaluation framework for SVC \\
Egilmez et al. [3] & & $\checkmark$ & $\checkmark$ & SDN controller for multimedia traffic \\
Ongaro et al. [15] & & $\checkmark$ & $\checkmark$ & Architecture based on SDN for multimedia applica- \\
& & & & tions \\
Zhao et al. [9] & $\checkmark$ & & $\checkmark$ & SDN controller for multiparty video conference \\
Yang et al. [10] & $\checkmark$ & $\checkmark$ & $\checkmark$ & Architecture based on SDN for SVC multicasting \\
\hline This manuscript & $\checkmark$ & $\checkmark$ & $\checkmark$ & Performance evaluation considering traditional IP \\
& & & & networks and SDN with scalable video coding \\
\hline
\end{tabular}

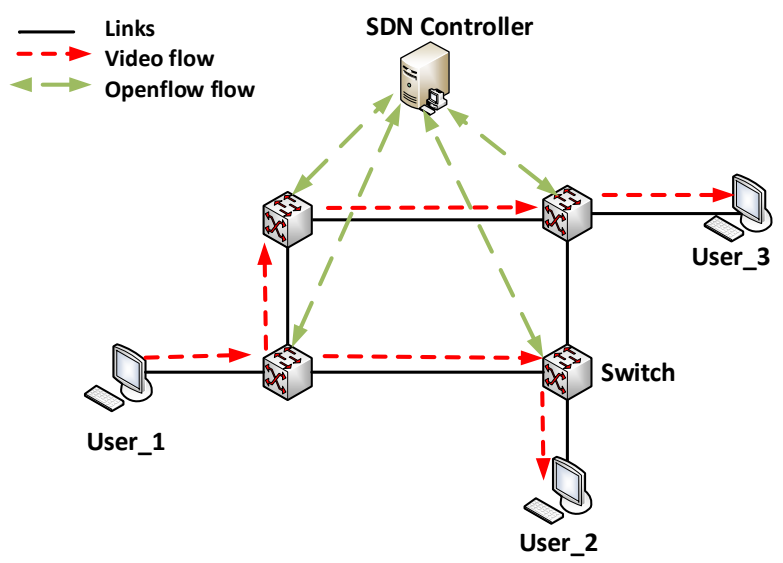

Figure 3. SDN video traffic

SDN decouples control and data planes, and it introduces the forwarding decision based on the flow and not only on the destination address (which is adopted in traditional IP networks). A flow represents packet sequences, and the packet fields are adopted in SDN for forwarding actions. Such an approach allows flexible traffic control, which is not limited by the capability of the tables implemented in switches/routers [4]. Openflow is a representative open protocol for communication between data and control planes [4].

Figure 3 depicts a video stream of a video conference in a SDN network. The video conferencing system is simpified as SDN controller replaces MCU and it also may allow efficient multiple transmissions as well as better traffic balance.

\section{Design of Experiments (DoE)}

To evaluate video conferencing systems with scalable video coding and software-defined networking, this work adopts a DoE [19] approach, considering a $l^{k}$ factorial design with $r$ replications. We have evaluated two factors $(k=2)$ with 2 levels $(l=2)$ : (i) network - non-SDN, SDN; and (ii) video non-scalable, SVC. Non-SDN denotes traditional IP network and non-scalable represents H.264/MPEG-4 AVC. 100 replications (samples) are adopted for each treatment to obtain mean values (with an approximate normal distribution) and to mitigate the influence of measurement noises (i.e., random errors).

The metrics of interest are throughput, delay, and peak signal to noise Ratio (PSNR). In this work, throughput takes into account the amount of data received (in Kbps) with a maximum delay of $300 \mathrm{~ms}$. Such a value is an important threshold assumed in many video conferencing systems [9] to allow conference smoothness. Delay takes into account the arrival time between packets (in milliseconds), also including packets that surpass the $300 \mathrm{~ms}$ threshold. PSNR is an objective metric (in decibels - $\mathrm{db}$ ) widely adopted for evaluating videos and still images [20][21]. It is a function of the mean square error between the original and received video frames and larger values indicate better video quality. Since PSNR requires the original video frames, it is considered a full reference technique. The experiments adopt mean PSNR [20], which is calculated averaging the PSNR values of all frames.

We have considered two distinct experiments, which contemplate single-path and multipath infrastructures to assess the respective impact on video conferencing systems. The infrastructure could be an explicit factor, but it would be a major source of variation in result analysis, and the overall measurement noise would prevent a finer comparison.

The following sections describe the adopted experiment setting and tools for the proposed evaluation.

\subsection{Experiment setting}

The infrastructures for single-path and multipath experiments take into account three machines, in which two machines are receivers and one machine is responsible for transmitting a video simulating the conference. The receivers differ from the adopted resolution, in which $176 \times 120$ resolution (Quarter Common Interchange Format - QCIF) represents a smartphone (Client_01) and $352 \times 240$ (Common Interchange Format - CIF) denotes a laptop computer (Client_02). These resolutions are commonly utilized by video conferencing systems [22]. Besides, all metrics are calculated considering data received by Client_01.

Figure 4 depicts the adopted single-path infrastructure, assuming a SDN network. The system adopts SDN controller, 


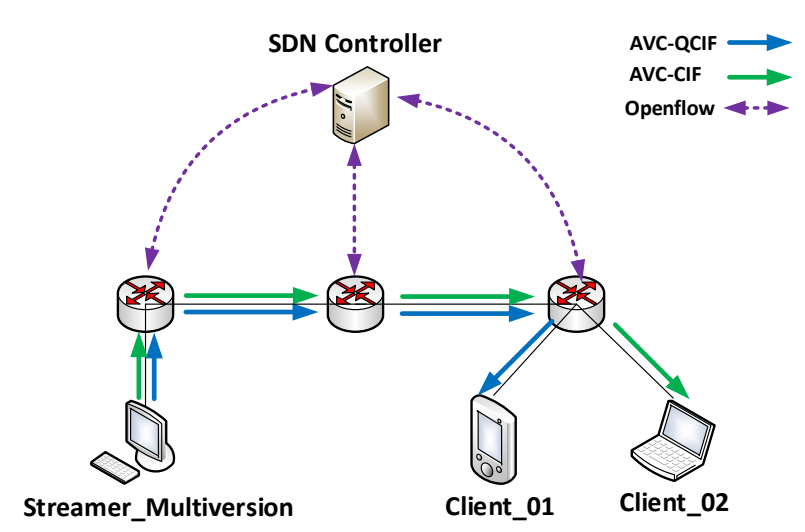

Figure 4. Multiversion video streaming in single-path infrastructure

which is responsible for forwarding the video streaming and it also performs actions to prioritize video traffic. Concerning non-SDN treatments, a simple MCU is utilized and it is main function is to forward the video streaming. Besides, such a figure also contemplates the simultaneous transmission of a multiversion video and the same infrastructure is also adopted for SVC streaming.

Figure 5 depicts the adopted infrastructure for multipath experiment, in which distinct paths are available between sender and receivers. This figure also assumes a SDN network and, in this case, a SDN controller replaces MCU. The controller is responsible for forwarding the video streaming and it also performs actions to prioritize video traffic. In this figure, a multilayer video (SVC) is adopted, which contemplates the video requirements for Client_01 and Client_02. The same infrastructure is adopted for non-SDN treatments, but a MCU is taken into account.

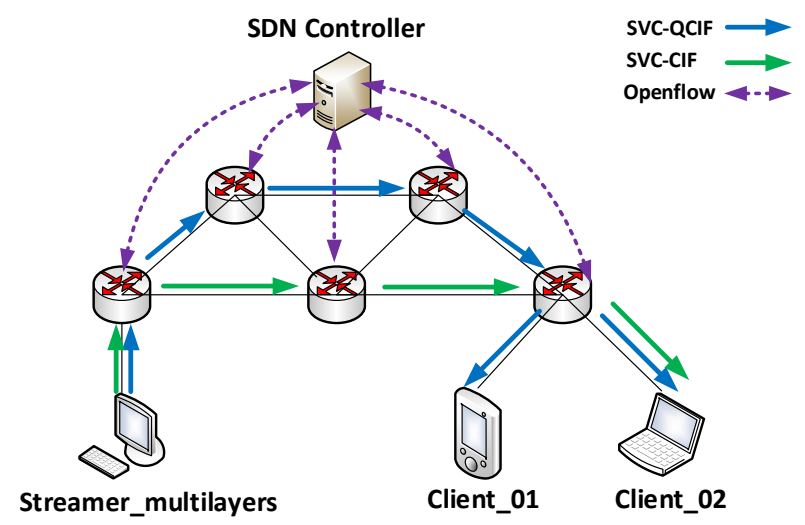

Figure 5. SVC streaming in multipath infrastructure

All links have $1 \mathrm{Mbps}$, and we insert a concurrent traffic contemplating $40 \%$ of link bandwidth to mimick a mildly congested network. In the single-path infrastructure, the concurrent traffic affects the whole network, but, considering the multipath infrastructure, the concurrent traffic contemplates the shortest path from streamer to Client_01 (which is the machine utilized to estimate the metrics of interest). A similar approach was adopted in [23] to allow the SDN controller to define a better path for the video streaming.

In this work, the rules of SDN controller transmit the video with highest resolution or the enhancement layers (for SVC) using the shortest path, which has the concurrent traffic. The lowest resolution video and base layer may be transmitted using other paths, as they are less congested and, thus, a video with minimal quality is guaranteed to be delivered. Besides, SDN network has a QoS policy, which automatically reserves $20 \%$ of link bandwidth for video transmission only when the video conference is carried out. This percentage is close to the bandwidth required to transmit the base layer and it is small enough to not impact other applications. Traditional networks could perform a similar approach based on traffic classification, but it has not been successfully adopted in this context [23]. SDN simplifies network programming for traffic control and it also allows a single network view, which facilitates the creation and management of QoS policies.

\subsection{Tools}

Mininet [4] has been utilized to construct a virtual environment for the adopted infrastructures, and the platform is executed in a machine with Intel Corel i5, 6GB RAM and operating system Ubuntu 16.04 .

Traditional IP network utilizes open shortest path first (OSPF) protocol, which, without loss of generality, takes the shortest path for delivering a packet [24]. For SDN network, the experiments adopt a SDN controller based on Ryu NOS[25], using the traffic rules explained in previous section. Traffic congestion is generated using D-ITG tool [26] and the generator is positioned in the switch closest to the streamer, such that only the shortest path is populated with concurrent traffic.

The experiments utilize a video ${ }^{1}$ which has a duration of 10 seconds. Such a video is enconded using non-scalable (AVC) and scalable (SVC) formats, and the sizes are 762KB and $790 \mathrm{~KB}$, respectively. SVC takes into account four layers, in which the base layer is also included. The evaluation framework for non-scalable video is Evalvid framework [13] and, for SVC, the experiments adopt SVEF framework [14].

Both frameworks utilize a similar evaluation process, which is depicted in Figure 6. The first step encodes the original video as scalable (SVC) or non-scalable (AVC) video. The video is then prepared to be sent as a trace, which includes additional information about each video frame. Next, the video is sent over the Mininet network. All packets received (by Client_01) are adopted to estimate delay, but only the packets received up to $300 \mathrm{~ms}$ (threshold) are taken into account to estimate throughput and to decode the final video. However, if a video frame is missing due to discarded packets, previously received frame is adopted to replace the lost frame (Frame Filler). This approach is carried out in order to keep the video

\footnotetext{
${ }^{1}$ Silent video - https://media.xiph.org/video/derf/
} 


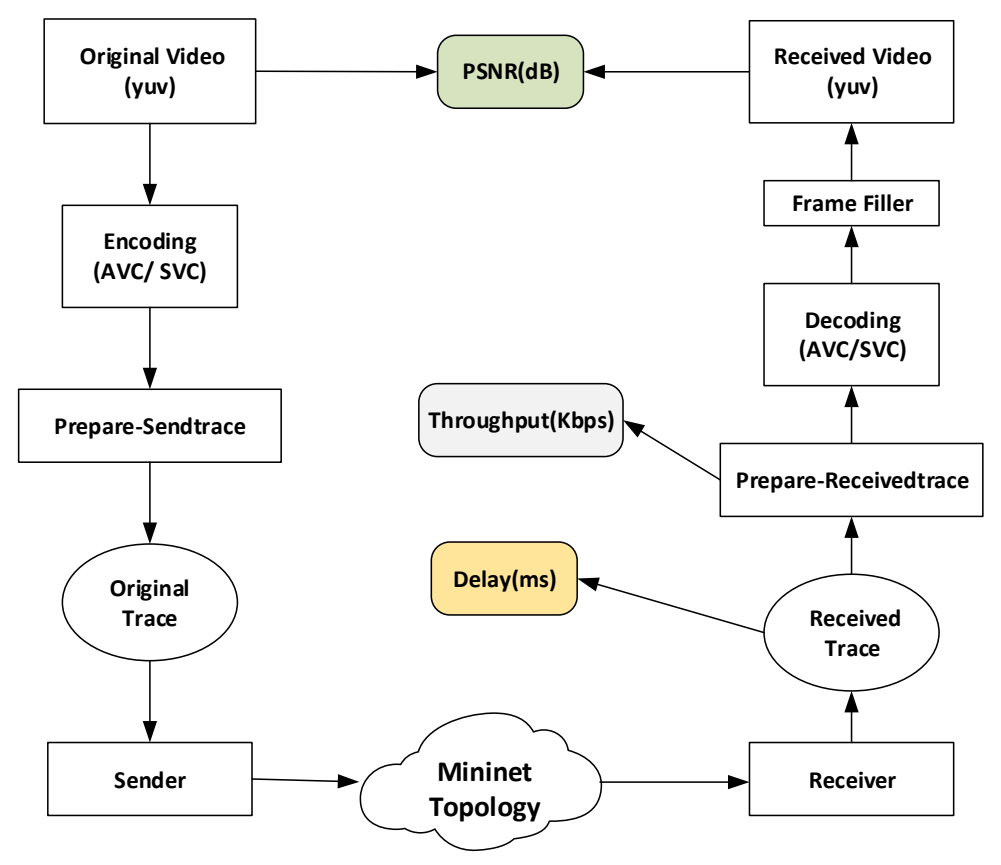

Figure 6. Evaluation process for video streaming

Table 2. ANOVA - Single-path experiment

\begin{tabular}{l|rrrr|rrrr|rrrr}
\hline \multicolumn{1}{c|}{ Source } & \multicolumn{4}{|c|}{ Throughput } & \multicolumn{4}{c|}{ Delay } & \multicolumn{4}{c}{ PSNR } \\
\hline \multirow{3}{*}{ Video } & Var.\% & df & F-Stat & P-value & Var.\% & df & F-Stat & P-value & Var.\% & df & F-Stat & P-value \\
\cline { 2 - 14 } & 1.40 & 1 & 9.72 & 0.002 & 12.13 & 1 & 135.82 & $<0.001$ & 81.76 & 1 & 16721.33 & $<0.001$ \\
Network & 52.38 & 1 & 364.13 & $<0.001$ & 52.52 & 1 & 587.96 & $<0.001$ & 11.91 & 1 & 2435.57 & $<0.001$ \\
Video*Network & 3.64 & 1 & 25.3 & $<0.001$ & 8.91 & 1 & 99.8 & $<0.001$ & 4.88 & 1 & 998.64 & $<0.001$ \\
Error & 42.58 & 296 & & & 26.44 & 296 & & & 1.45 & 296 & & \\
\hline
\end{tabular}

with the same amount of frames as the original, such that mean PSNR can be calculated.

\section{Experimental results}

As discussed in Section 4, two distinct experiments are carried out. The first experiment contemplates a network infrastructure with a single path between devices, and the second experiment adopts a multipath infrastructure. For the sake of organization, the first experiment is denominated single-path experiment and the second experiment is named as multipath experiment.

Results are presented using ANOVA analysis (which statistically assesses each factor), and they are described in Table 2 and 3. In these tables, column Source describes the sources of variation for each metric, which include the factors video type (Video), network (Nework), and their interaction (e.g., Video.*Network. Error is also a source of variation that may represent noise in the measurements or variation that cannot be explained by the adopted factors [19]. Other columns represent the following items: var.\% denotes the impact of each factor on metric variation; $d f$ is the degree of freedom; and $F-$ stat. is the F statistic with the respective $p-v a l u e$.
Next sections discuss each experiment, also taking into account the outcome obtained with Tukey's procedure (a postANOVA test) [19]. Besides, Figure 7 and 8 depict mean values with $95 \%$ confidence intervals.

\subsection{Single-path experiment}

Table 2 depicts results for the single-path experiment, which indicates all factors are statically significant for all metrics (i.e., $p$-value $<0.05$ ). Factor Network is the main source of variation for throughput and delay, whereas factor video is most responsible for the variation on PSNR.

Figure 7 depicts the results for all metrics. Concerning non-SDN network, there is no statistical difference for throughput (Figure 7 (a)) and delay (Figure 7 (b)), taking into account distinct video types. In the adopted infrastructure, traditional IP network does not have dynamic QoS rules to deal with concurrent traffic and video stream, making the delay very high. Since the threshold is also not met for many packets, the throughput is very low. Nevertheless, SDN network positively impacts a video conference with SVC, such that a $7 \mathrm{x}$ increase in throughput is obtained. Concerning non-scalable video and throughput, SDN is $2 \mathrm{x}$ better than a 


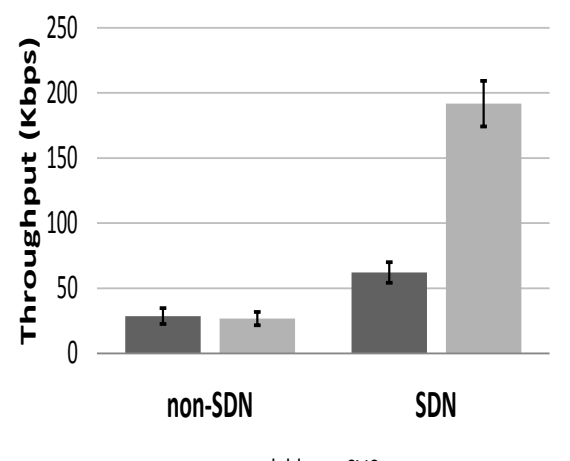

non-scalable $\quad$ SVC

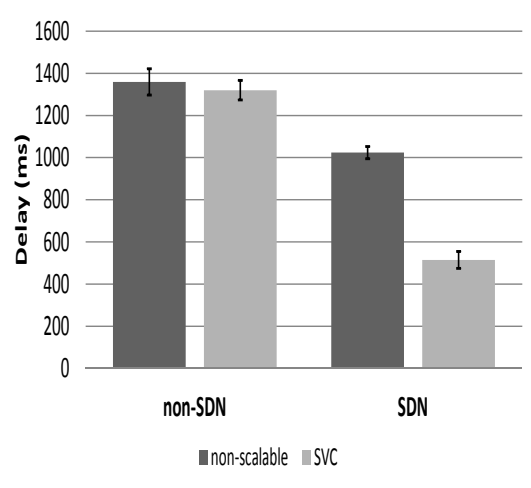

(b)

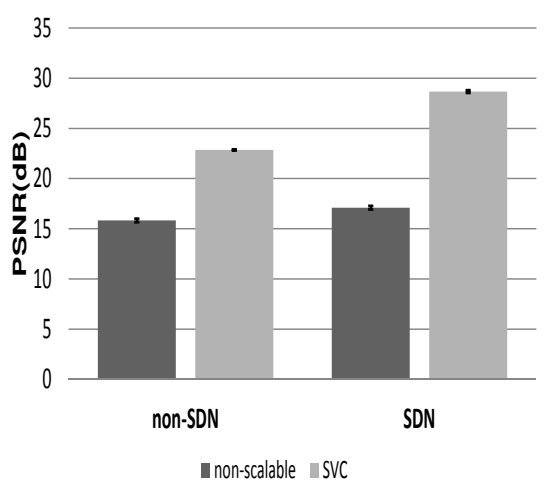

(c)

(a)

Figure 7. Single-path experiment: throughput (a), delay (b) and PSNR (c)

Table 3. ANOVA - Multipath experiment

\begin{tabular}{|c|c|c|c|c|c|c|c|c|c|c|c|c|}
\hline Source & \multicolumn{4}{|c|}{ Throughput } & \multicolumn{4}{|c|}{ Delay } & \multicolumn{4}{|c|}{ PSNR } \\
\hline \multirow[b]{2}{*}{ Video } & Var.\% & $\mathrm{df}$ & F-Stat & P-value & Var.\% & $\mathrm{df}$ & F-Stat & P-value & Var.\% & $\mathrm{df}$ & F-Stat & P-value \\
\hline & 1.00 & 1 & 6.94 & 0.0 & 6.27 & 1 & 132 & $<0$ & 69.23 & 1 & 5536.66 & $<0.001$ \\
\hline Network & 54.43 & 1 & 362.82 & $<0.001$ & 75.48 & 1 & 1589.58 & $<0.001$ & 19.61 & 1 & 1568.37 & $<0.001$ \\
\hline Video*Network & 3.78 & 1 & 26.19 & $<0.001$ & 4.19 & 1 & 88.38 & $<0.001$ & 7.46 & 1 & 596.86 & $<0.001$ \\
\hline Error & 296 & 42.78 & & & 296 & 14.06 & & & 296 & 3.70 & & \\
\hline
\end{tabular}

traditional network, since SDN assures a minimum bandwidth (of 20\%) when a video conference streaming is detected.

Concerning video quality, the highest value is obtained with SVC, as the threshold (300ms) only impacts the enhancement layers. However, non-scalable video is also benefited with SDN due to the adopted traffic rules.

\subsection{Multipath experiment}

Table 3 shows the results for the multipath experiment, which indicate all factors are also statically significant for all metrics $(p-$ value $<0.05)$. Similar to single-path experimment, factor Network is the major source of variation for throughput and delay, and PSNR is most impacted by video type.

Figure 8 depicts the values for all metrics. Regarding throughput, Figure 8 (a) shows the values are very low in nonSDN network and it also indicates no statistical difference for video type. Nevertheless, SDN network has an important role in SVC transmission, as a 6x increase in throughput is obtained. Concerning non-scalable video, SDN also provides improvement in throughput, since SDN takes benefit from the multipaths provided by the infrastructure.

As a consequence, delay (Figure 8 (b)) is very high for nonSDN network due to the concurrent traffic in the shortest path and the values are not statistically different for non-scalable and scalable videos. On the other hand, SDN reduces delay due to dynamic rules to deal with congested links and the QoS policy that reserves bandwidth for video conference. Nonscalable video is transmitted 33\% faster and SVC transmission is also considerably improved (almost $3 x$ faster than non-SDN network), since the base layers are not transmitted using the (congested) shortest path. Comparing SVC and non-scalable video in SDN network, SVC is almost 50\% faster.

Video quality has the highest value with SVC, as the threshold (300ms) does not significantly affect the base layer. Besides, SDN still provides benefits to non-scalable video, since QoS policy allows improvement on video transmission.

\subsection{General remarks}

Results have been obtained considering a bandwidth constraint due to the insertion of concurrent traffic. Assuming a congestion-free network, all results would not be statistically different, as the adopted threshold would be met. On the other hand, in a very congested non-SDN network, the delays would be very high, leading to an unfeasible video conference. As demonstrated, SDN can better deal with such a situation due to QoS rules based on packet flow and the single network view.

Besides, SVC provides a remarkable feature, which is the adoption of a base layer that allows the video be smoothly playable without additional data. Enhancement layers provide better video quality, but their reception does not fully impact the conference. For instance, throughput and delay are not statically different for non-SDN networks, but PSNR is considerably better for SVC videos as they can be smoothly played. As indicated by experimental results, the combination of SVC and SDN provides the best results and it seems very promising for dealing with device heterogeneity, distinct network infrastructures and concurrent traffic. 


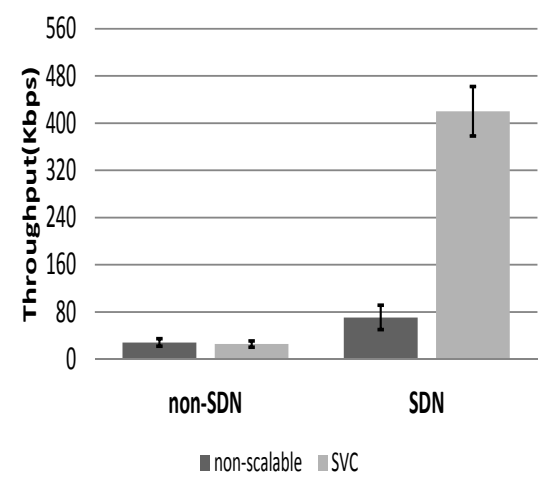

(a)

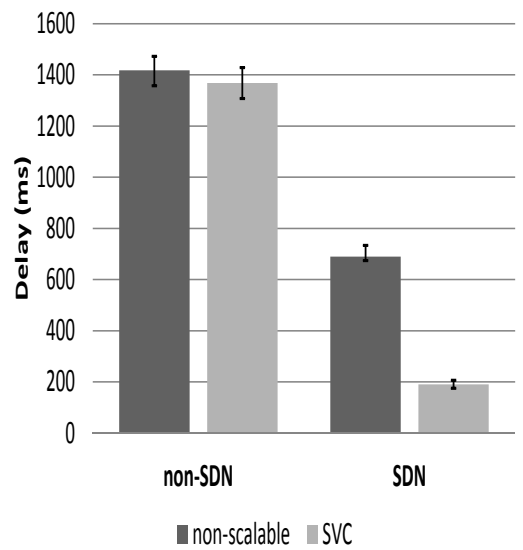

(b)

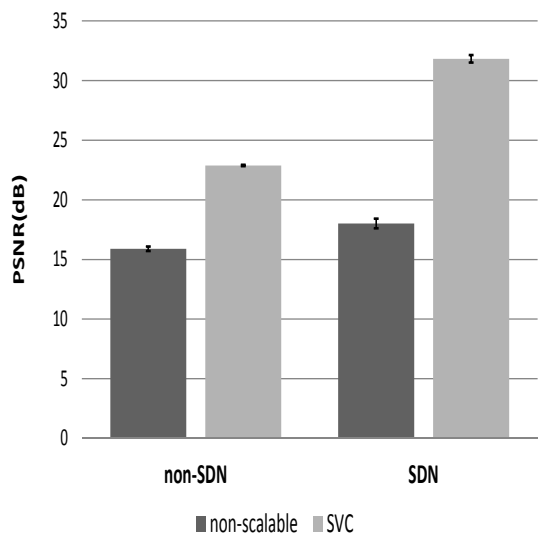

(c)

Figure 8. Multipath experiment: throughput (a), delay (b) and PSNR (c)

\section{Conclusion}

Device heterogeneity is a representative issue in video conferencing systems, and scalable video coding and softwaredefined networking are technologies that can assist in meeting the conference requirements imposed by distinct devices. Despite the importance of these technologies, few works evaluate the peformance of video conferencing systems taking into account SVC and SDN concomitantly.

This paper presents a performance evaluation of video conferencing systems, taking into account the following factors: (i) network (traditional IP and SDN); and (ii) video (non-scalable and SVC). Experiments have been carried out also considersing network infrastructures with single path and multiple paths. Results demonstrate SDN in conjuction with SVC can provide better quality of experience, since throughput, packet delay and PSNR are considerably improved.

As future works, we are planning to create analytic models to assess different architectures for video conferencing systems on SDN networks.

\section{Author contributions}

Francisco Oliveira - Work Conception and Experiments

Eduardo Tavares, Erica Sousa, Bruno nogueira - Statistical Analysis

\section{References}

[1] CISCO. The Zettabyte Era: Trends and Analysis. San Jose, CA, USA, 2016.

[2] THOM, G. H.323: the multimedia communications standard for local area networks. IEEE Comm. Mag., v. 34, n. 12, p. 52-56, 1996.

[3] EGILMEZ, H. E. et al. Openqos: An openflow controller design for multimedia delivery with end-to-end quality of service over software-defined networks. In: KUO, C.-C. J.; NARAYANAN, S.; ORTEGA, A. (Ed.). Proceedings of The 2012 Asia Pacific Signal and Information Processing Association Annual Summit and Conference. Hollywood, California, USA: APSPPAAAC, 2012. v. 1.

[4] KREUTZ, D. et al. Software-defined networking: A comprehensive survey. Proceedings IEEE, v. 103, n. 1, p. 14-76, 2015.

[5] OPEN NETWORKING FOUNDATION. Softwaredefined networking: The new norm for networks. Menlo Park, CA, USA, 2012.

[6] MCKEOWN, N. et al. Openflow: Enabling innovation in campus networks. SIGCOMM Comput. Commun. Rev., v. 38, n. 2, p. 69-74, 2008.

[7] SCHWARZ, H.; MARPE, D.; WIEGAND, T. Overview of the scalable video coding extension of the h.264/avc standard. IEEE Trans. Circuits Syst. Video Technol, v. 17, n. 9, p. 1103-1120, 2007.

[8] XU, Y. et al. Video telephony for end-consumers: Measurement study of google+, ichat, and skype. In: BYERS, J.; KUROSE, J. (Ed.). Proceedings of the 2012 Internet Measurement Conference. New York, NY, USA: ACM, 2012. (IMC'12, v. 1).

[9] ZHAO, M.; AL et. Software defined network-enabled multicast for multi-party video conferencing systems. In: SAFAEI, F. (Ed.). 2014 IEEE International Conference on Communications (ICC). Sydney, Australia: IEEE, 2014. (1, v. 1).

[10] YANG, E.-Z. et al. A video conferencing system based on sdn-enabled svc multicast. Front Inform Tech El, v. 17, n. 7, p. 672-681, 2016.

[11] MCCANNE, S.; JACOBSON, V.; VETTERLI, M. Receiver-driven layered multicast. SIGCOMM Comput. Commun. Rev., v. 26, n. 4, p. 117-130, 1996. 
[12] CASTELLANOS, W. E.; GUERRI, J. C.; ARCE, P. Svceval-ra: an evaluation framework for adaptive scalable video streaming. Multmed. Tools and Applications, v. 76, n. 1, p. 437-461, 2017.

[13] KLAUE, J.; RATHKE, B.; WOLISZ, A. Evalvid - a framework for video transmission and quality evaluation. In: KEMPER, P.; SANDERS, W. H. (Ed.). Computer Performance Evaluation. Modelling Techniques and Tools: 13th International Conference. Urbana, IL, USA: Springer, 2003. v. 13.

[14] DETTI, A.; AL et. Svef: an open-source experimental evaluation framework for h.264 scalable video streaming. In: ELMAGHRABY, A. S. (Ed.). 2009 IEEE Symposium on Computers and Communications. Sousse, Tunisia: IEEE, 2009. (1, v. 1).

[15] ONGARO, F. et al. Enhancing the quality level support for real-time multimedia applications in software-defined networks. In: GERLA, M.; SU, G.-M. (Ed.). 2015 International Conference on Computing, Networking and Communications. Garden Grove, CA, USA: IEEE, 2015. (1, v. 1).

[16] RODRÍGUEZ, P. et al. Materialising a new architecture for a distributed mcu in the cloud. Comput. Stand. \& Interfaces, v. 44, n. 1, p. 234-242, 2016.

[17] PURI, A.; AL. et. Video coding using the h.264/mpeg-4 avc compression standard. Sig. Proc. Img. Comm., v. 19, n. 9, p. 793-849, 2004.
[18] WIEN, M.; SCHWARZ, H.; OELBAUM, T.

Performance analysis of svc. IEEE Trans. Circuits Syst., v. 17, n. 9, p. 1194-1203, 2007.

[19] MONTGOMERY, D. C.; RUNGER, G. C. Applied statistics and probability for engineers. 6. ed. Hoboken, New Jersey, USA: Wiley, 2013. v. 1. (1, v. 1).

[20] PULLANO, V.; AL. et. Psnr evaluation and alignment recovery for mobile satellite video broadcasting. In:

CORAZZA, G.; SCALISE, S.; VANELLI-CORALLI, A. (Ed.). Advanced Satellite Multimedia Systems Conference (ASMS). Baiona, Spain: IEEE, 2012. (1, v. 1).

[21] ITU. Methodology for the subjective assessment of the quality of television pictures BT Series Broadcasting service. 2012.

[22] BING, B. Next-Generation Video Coding and Streaming. 1. ed. Hoboken, New Jersey, USA: Wiley, 2015. v. 1. (1, v. 1). [23] EGILMEZ, H. E.; TEKALP, A. M. Distributed qos architectures for multimedia streaming over software defined networks. IEEE Trans. Multmed., v. 16, n. 6, p. 1597-1609, 2014.

[24] JAKMA, P.; LAMPARTER, D. Introduction to the quagga routing suite. IEEE Netw., v. 28, n. 2, p. 42-48, 2014. [25] MORITA, K.; YAMAHATA, I. Ryu Network Operating System. Toyko, Japan, 2016.

[26] BOTTA, A.; DAINOTTI, A.; PESCAPÈ, A. A tool for the generation of realistic network workload for emerging networking scenarios. Comput. Netw., v. 56, n. 15, p. 3531-3547, 2012. 